\title{
Length functions of Hitchin representations
}

\author{
GUILLAUME DREYER
}

\begin{abstract}
Given a Hitchin representation $\rho: \pi_{1}(S) \rightarrow \operatorname{PSL}_{n}(\mathbb{R})$, we construct $n$ continuous functions $\ell_{i}^{\rho}: \mathcal{C}^{\text {Höl }}(S) \rightarrow \mathbb{R}$ defined on the space of Hölder geodesic currents $\mathcal{C}^{\text {Höl }}(S)$ such that, for a closed, oriented curve $\gamma$ in $S$, the $i^{\text {th }}$ eigenvalue of the matrix $\rho(\gamma) \in \operatorname{PSL}_{n}(\mathbb{R})$ is of the form $\pm \exp \ell_{i}^{\rho}(\gamma)$ : such functions generalize to higher rank Thurston's length function of Fuchsian representations. Identities and differentiability properties of these lengths $\ell_{i}^{\rho}$, as well as applications to eigenvalue estimates, are also considered.
\end{abstract}

57M50; 57M05, 37F30, 22E40

Let $S$ be a closed, connected, oriented surface $S$ of genus $g \geq 2$. This article is concerned with homomorphisms $\rho: \pi_{1}(S) \rightarrow \mathrm{PSL}_{n}(\mathbb{R})$ from the fundamental group $\pi_{1}(S)$ to the Lie group $\operatorname{PSL}_{n}(\mathbb{R})$ (equal to the special linear group $\operatorname{SL}_{n}(\mathbb{R})$ if $n$ is odd, and to $\mathrm{SL}_{n}(\mathbb{R}) /\{ \pm \mathrm{Id}\}$ if $n$ is even), and more precisely with elements lying in Hitchin components $\operatorname{Hit}_{n}(S)$ of the $\mathrm{PSL}_{n}(\mathbb{R})$-character variety

$$
\mathcal{R}_{\mathrm{PSL}_{n}(\mathbb{R})}(S)=\operatorname{Hom}\left(\pi_{1}(S), \mathrm{PSL}_{n}(\mathbb{R})\right) / / \mathrm{PSL}_{n}(\mathbb{R})
$$

identified by N Hitchin [18]. Here, the "double bar" sign indicates that the precise definition of the character variety $\mathcal{R}_{\mathrm{PSL}_{n}(\mathbb{R})}(S)$ requires that the quotient be taken in the sense of geometric invariant theory (Mumford, Fogarty and Kirwan [21]); however, for the component $\operatorname{Hit}_{n}(S)$ that we are interested in, this quotient construction coincides with the usual topological quotient.

A Hitchin component $\operatorname{Hit}_{n}(S)$ is defined as a component of $\mathcal{R}_{\mathrm{PSL}_{n}(\mathbb{R})}(S)$ that contains some $n-F u c h s i a n$ representation, namely some homomorphism $\rho: \pi_{1}(S) \rightarrow \operatorname{PSL}_{n}(\mathbb{R})$ of the form

$$
\rho=\iota \circ r
$$

where: $r: \pi_{1}(S) \rightarrow \mathrm{PSL}_{2}(\mathbb{R})$ is a discrete, injective homomorphism, and

$$
\iota: \mathrm{PSL}_{2}(\mathbb{R}) \rightarrow \mathrm{PSL}_{n}(\mathbb{R})
$$

is the preferred homomorphism defined by the $n$-dimensional, irreducible representation of $\mathrm{SL}_{2}(\mathbb{R})$ into $\mathrm{SL}_{n}(\mathbb{R})$. These components $\mathrm{Hit}_{n}(S)$ were singled out by 
$\mathrm{N}$ Hitchin [18] who first suggested the interest in studying their elements. We shall refer to elements of $\operatorname{Hit}_{n}(S)$ as Hitchin representations.

Motivations for studying Hitchin representations find their origin in the case where $n=2$. Hitchin components $\mathrm{Hit}_{2}(S)$ then coincide with Teichmüller components $\mathcal{T}(S)$ of $\mathcal{R}_{\mathrm{PSL}_{2}(\mathbb{R})}(S)$, whose elements, known as Fuchsian representations, are of particular interest as they correspond to conjugacy classes of holonomies of marked hyperbolic structures on the surface $S$. In addition, every element of $\mathcal{T}(S)$ is a discrete, injective homomorphism, and reversely, any such homomorphism lies in some component $\mathcal{T}(S)$ (Weil [26] and Margulis [20]). It is a result due to W Goldman [13] that $\mathcal{R}_{\mathrm{PSL}_{2}(\mathbb{R})}(S)$ possesses exactly two Teichmüller components $\mathcal{T}(S)$; each of these components $\mathcal{T}(S)$ is known to be homeomorphic to $\mathbb{R}^{6 g-6}$ (Thurston [25] and Fathi, Laudenbach and Poénaru [10]).

In his foundational paper, Hitchin [18] proved that, in the case where $n \geq 3$, there are one or two Hitchin components $\operatorname{Hit}_{n}(S)$ in $\mathcal{R}_{\mathrm{PSL}_{n}(\mathbb{R})}(S)$ according to whether $n$ is odd or even, and a beautiful result of Hitchin is that each of these components $\mathrm{Hit}_{n}(S)$ is homeomorphic to $\mathbb{R}^{(2 g-2)\left(n^{2}-1\right)}$. Hitchin's proof is based the theory of Higgs bundles, and as observed by Hitchin, this geometric analysis framework offers no information about the geometry of the elements of $\operatorname{Hit}_{n}(S)$. The first geometric result about Hitchin representations is to due to S Choi and W Goldman [8] who showed that, for $n=3$, the Hitchin component $\mathrm{Hit}_{3}(S)$ parametrizes the deformation space of real convex projective structures on the surface $S$. As a consequence of their work, they showed the faithfulness and the discreetness for the elements of $\operatorname{Hit}_{3}(S)$.

About a decade ago, F Labourie [19] (see also Guichard [16] and Guichard and Wienhard [17]) proved the following result.

Theorem 1 (Labourie [19]) Let $\rho: \pi_{1}(S) \rightarrow \operatorname{PSL}_{n}(\mathbb{R})$ be a Hitchin representation. Then $\rho$ is discrete and injective. In addition, the image $\rho(\gamma) \in \operatorname{PSL}_{n}(\mathbb{R})$ of any nontrivial $\gamma \in \pi_{1}(S)$ is diagonalizable, its eigenvalues are all real with distinct absolute values.

The above statement comes as a consequence (among others) of a remarkable Anosov property for Hitchin representations discovered by Labourie [19]. More precisely, let $\rho: \pi_{1}(S) \rightarrow \operatorname{PSL}_{n}(\mathbb{R})$ be a Hitchin representation that lifts to $\rho: \pi_{1}(S) \rightarrow \operatorname{SL}_{n}(\mathbb{R})$; consider the flat, twisted $\mathbb{R}^{n}$-bundle $T^{1} S \times_{\rho} \mathbb{R}^{n}=T^{1} S \times \mathbb{R}^{n} / \pi_{1}(S) \rightarrow T^{1} S$, where $T^{1} S$ is the unit tangent bundle of $S$; let $\left(G_{t}\right)_{t \in \mathbb{R}}$ on $T^{1} S \times{ }_{\rho} \mathbb{R}^{n}$ be the flow that lifts the geodesic flow $\left(g_{t}\right)_{t \in \mathbb{R}}$ on $T^{1} S$ via the flat connection. The total space $T^{1} S \times{ }_{\rho} \mathbb{R}^{n}$ splits as a sum of line subbundles $V_{1} \oplus \cdots \oplus V_{n}$ with the property that each line subbundle $V_{i} \rightarrow T^{1} S$ is invariant under the action of the flow $\left(G_{t}\right)_{t \in \mathbb{R}}$. In addition, 
the action of the flow $\left(G_{t}\right)_{t \in \mathbb{R}}$ is Anosov in the following sense: pick a Riemannian metric \| $\|$ on $T^{1} S \times_{\rho} \mathbb{R}^{n} \rightarrow T^{1} S$; there exist some constants $A \geq 0$ and $a>0$ such that, for every $u \in T^{1} S$, for every unit vectors $X_{i}(u) \in V_{i}(u)$ and $X_{j}(u) \in V_{j}(u)$, for every $t>0$,

$$
A e^{-a t} \geq \begin{cases}\frac{\left\|G_{t} X_{j}(u)\right\|_{g_{t}(u)}}{\left\|G_{t} X_{i}(u)\right\|_{g_{t}(u)}} & \text { if } i>j \\ \frac{\left\|G_{-t} X_{j}(u)\right\|_{g_{-t}(u)}}{\left\|G_{-t} X_{i}(u)\right\|_{g_{-t}(u)}} & \text { if } i<j .\end{cases}
$$

\section{Results}

Given a Hitchin representation $\rho: \pi_{1}(S) \rightarrow \operatorname{PSL}_{n}(\mathbb{R})$, our main result uses Labourie's dynamical framework to define a family of $n$ length functions $\ell_{i}^{\rho}$ associated to $\rho$; these length functions extend to Hitchin representations Thurston's length function of Fuchsian representations in the Teichmüller space $\mathcal{T}(S)$.

Thurston [25] considers the space of measured laminations $\mathcal{M L}(S)$ of $S$, that is a certain completion of the set of all isotopy classes of simple, closed, unoriented curves in $S$. He then associates to a Fuchsian representation $r: \pi_{1}(S) \rightarrow \mathrm{PSL}_{2}(\mathbb{R})$ in $\mathcal{T}(S)$ a continuous, homogeneous function $\ell^{r}: \mathcal{M L}(S) \rightarrow \mathbb{R}$ such that, for every simple, closed, unoriented curve $\gamma \subset S$,

$$
\ell^{r}(\gamma)=\frac{1}{2} \log \left|\lambda_{1}^{r}(\gamma)\right|,
$$

where $\left|\lambda_{1}^{r}(\gamma)\right|$ is the largest absolute value of the eigenvalues of $r(\gamma) \in \operatorname{PSL}_{2}(\mathbb{R})$. Geometrically, $r: \pi_{1}(S) \rightarrow \mathrm{PSL}_{2}(\mathbb{R})$ is the holonomy of a marked hyperbolic structure $m$ on the surface $S$; the number $\ell^{r}(\gamma)$ is then the length of the unique, simple, closed, unoriented $m$-geodesic in $S$ that is freely homotopic to the simple, closed, unoriented curve $\gamma$. This length function $\ell^{r}: \mathcal{M L}(S) \rightarrow \mathbb{R}$ has proved to be a fundamental tool in the study of 2 and 3-dimensional hyperbolic manifolds.

Thurston's length function $\ell^{r}$ was later extended by F Bonahon $[1 ; 2]$ to the larger space of measure geodesic currents $\mathcal{C}(S)$ of $S$, which is a certain completion of the set of all isotopy classes of closed, oriented curves in $S$. Later, Bonahon [3] also developed a differential calculus for measured laminations, that is based on Hölder geodesic currents. In particular, he obtains differentiability properties for Thurston's original function $\ell^{r}: \mathcal{M L}(S) \rightarrow \mathbb{R}$ by continuously extending $\ell^{r}: \mathcal{M L}(S) \rightarrow \mathbb{R}$ to the space of Hölder geodesic currents $\mathcal{C}^{\text {Höl }}(S)$ of $S$.

We generalize these constructions in the case where $\rho: \pi_{1}(S) \rightarrow \operatorname{PSL}_{n}(\mathbb{R})$ is a Hitchin representation. Let $\gamma \in \pi_{1}(S)$ be nontrivial element; by Theorem 1, the eigenvalues 
$\lambda_{i}^{\rho}(\gamma)$ of the matrix $\rho(\gamma) \in \mathrm{PSL}_{n}(\mathbb{R})$ can be indexed so that

$$
\left|\lambda_{1}^{\rho}(\gamma)\right|>\left|\lambda_{2}^{\rho}(\gamma)\right|>\cdots>\left|\lambda_{n}^{\rho}(\gamma)\right| .
$$

Theorem 2 (Length functions) Let $\rho: \pi_{1}(S) \rightarrow \mathrm{PSL}_{n}(\mathbb{R})$ be a Hitchin representation, and let $\mathcal{C}^{\text {Höl }}(S)$ be the vector space of Hölder geodesic currents. For every $i=1$, $2, \ldots, n$, there exists a continuous, linear function

$$
\ell_{i}^{\rho}: \mathcal{C}^{\mathrm{Höl}}(S) \rightarrow \mathbb{R}
$$

such that, for every closed, oriented curve $\gamma \subset S, \ell_{i}^{\rho}(\gamma)=\log \left|\lambda_{i}^{\rho}(\gamma)\right|$. This continuous extension is unique on the space of measure geodesic currents $\mathcal{C}(S) \subset \mathcal{C}^{\mathrm{Höl}}(S)$.

In addition, let $\mathfrak{R}: T^{1} S \rightarrow T^{1} S$ be the orientation reversing involution, namely $\mathfrak{R}$ is the map defined by $\Re(u)=-u$, where $u \in T_{x}^{1} S$. For every Hölder geodesic current $\alpha \in \mathcal{C}^{\text {Höl }}(S), \mathfrak{R}^{*} \alpha$ is the pullback current of $\alpha$ under the involution $\mathfrak{R}$.

Theorem 3 (Identities) For every Hölder geodesic current $\alpha \in \mathcal{C}^{\text {Höl }}(S)$ :

(i) $\sum_{i=1}^{n} \ell_{i}^{\rho}(\alpha)=0$

(ii) $\ell_{i}^{\rho}\left(\Re^{*} \alpha\right)=-\ell_{n-i+1}^{\rho}(\alpha)$

The above two identities are suggested by the case where $\alpha \in \mathcal{C}^{\text {Höl }}(S)$ is a closed, oriented curve $\gamma \in \pi_{1}(S)$. Indeed, since $\rho(\gamma) \in \operatorname{PSL}_{n}(\mathbb{R}), \sum_{i=1}^{n} \log \left|\lambda_{i}^{\rho}(\gamma)\right|=0$. Moreover, as a consequence of our indexing conventions, $\lambda_{i}^{\rho}\left(\gamma^{-1}\right)=1 / \lambda_{n-i+1}^{\rho}(\gamma)$, and thus $\log \left|\lambda_{i}^{\rho}\left(\gamma^{-1}\right)\right|=-\log \left|\lambda_{n-i+1}^{\rho}(\gamma)\right|$.

The continuity property of Theorem 2 is the fundamental feature of the length functions $\ell_{i}^{\rho}: \mathcal{C}^{\mathrm{Höl}}(S) \rightarrow \mathbb{R}$. As an application of this continuity, we prove the two following results; the proofs use the full force of Hölder geodesic currents.

Theorem 4 (Tangentiability) The functions $\ell_{i}^{\rho}: \mathcal{C}^{\text {Höl }}(S) \rightarrow \mathbb{R}$ restrict to functions $\ell_{i \mid \mathcal{M L}(S)}^{\rho}: \mathcal{M L}(S) \rightarrow \mathbb{R}$ that are tangentiable, namely, if $\left(\alpha_{t}\right)_{t \geq 0} \subset \mathcal{M L}(S)$ is a smooth 1-parameter family of measured laminations with tangent vector $\dot{\alpha_{0}}=\left.\frac{d}{d t^{+}} \alpha_{t}\right|_{t=0}$ at $\alpha_{0}$, then

$$
\left.\frac{d}{d t^{+}} \ell_{i}^{\rho}\left(\alpha_{t}\right)\right|_{t=0}=\ell_{i}^{\rho}\left(\dot{\alpha}_{0}\right)
$$

Finally, we prove the following asymptotic estimate for the eigenvalues of a Hitchin representation. 
Theorem 5 (Eigenvalue estimate) Let $\rho: \pi_{1}(S) \rightarrow \mathrm{PSL}_{n}(\mathbb{R})$ be a Hitchin representation, and let $\alpha, \beta \in \pi_{1}(S)$. For every $i=1, \ldots, n$, the ratio

$$
\frac{\lambda_{i}^{\rho}\left(\alpha^{m} \beta\right)}{\lambda_{i}^{\rho}(\alpha)^{m}}
$$

has a finite limit as $m$ tends to $\infty$. This limit is equal to $e^{\ell_{i}^{\rho}(\dot{\alpha})}$ for a certain Hölder geodesic current $\dot{\alpha} \in \mathcal{C}^{\text {Höl }}(S)$.

\section{Remarks}

Dreyer [9] extends to Hitchin representations Thurston's cataclysm deformations, which themselves generalize (left) earthquake deformations of hyperbolic structures on surfaces (Thurston [24; 25]). Given a Hitchin representation $\rho: \pi_{1}(S) \rightarrow \operatorname{PSL}_{n}(\mathbb{R})$, we study various geometric aspects of these cataclysms, and prove a variational formula for the associated length functions $\ell_{i}^{\rho}$.

Another motivation for introducing length functions associated to a Hitchin representation is part of the development of a new system of coordinates on Hitchin components $\operatorname{Hit}_{n}(S)$. In [18], Hitchin showed that $\operatorname{Hit}_{n}(S)$ is diffeomorphic to $\mathbb{R}^{(2 g-2)\left(n^{2}-1\right)}$; his parametrization is based on Higgs bundle techniques, and in particular requires the initial choice of a complex structure on $S$. In joint work with F Bonahon [5; 6], we construct a geometric, real analytic parametrization of Hitchin components $\mathrm{Hit}_{n}\left(\mathbb{R}^{n}\right)$. One feature of this parametrization is that it is based on topological data only. In essence, our coordinates are an extension of Thurston's shearing coordinates (Thurston [24] and Bonahon [1]) on the Teichmüller space $\mathcal{T}(S)$, combined with Fock-Goncharov coordinates [11] on moduli spaces of positive, framed, local systems on a punctured surface. In particular, the length functions $\ell_{i}^{\rho}$ play a crucial role in analyzing the image of this parametrization.

\section{Acknowledgments}

I would like to thank my advisor, Francis Bonahon, for his invaluable guidance and support. I also thank the referee for his careful reading of the first draft of this article along with numerous remarks and suggestions. This research was partially supported by the grant DMS-0604866 from the National Science Foundation.

\section{The eigenbundles of a Hitchin representation}

Our construction makes great use of the machinery developed in Labourie [19], we thus begin with reviewing some of Labourie's framework. It is convenient to endow 
the surface $S$ with an arbitrary hyperbolic metric $m_{0}$. It induces a $m_{0}$-geodesic flow $\left(g_{t}\right)_{t \in \mathbb{R}}$ on the unit tangent bundle $T^{1} S$ of $S$; we refer to the associated orbit space as the $m_{0}$-geodesic foliation $\mathcal{F}$ of $T^{1} S$.

Let $\rho: \pi_{1}(S) \rightarrow \mathrm{PSL}_{n}(\mathbb{R})$ be a Hitchin representation. Since $\rho$ lies in the same component as some $n$-Fuchsian representation, it lifts to a representation valued in $\mathrm{SL}_{n}(\mathbb{R})$, that we still denote by $\rho: \pi_{1}(S) \rightarrow \mathrm{SL}_{n}(\mathbb{R})$; see Goldman [13] for details. Consider the flat twisted $\mathbb{R}^{n}$-bundle

$$
T^{1} S \times \mathbb{R}^{n}=T^{1} \widetilde{S} \times \mathbb{R}^{n} / \pi_{1}(S),
$$

where $\widetilde{S}$ is the universal cover of $S$, and where the action of $\pi_{1}(S)$ is defined by the property that, for every $\gamma \in \pi_{1}(S)$, for every $(u, X) \in T^{1} \widetilde{S} \times \mathbb{R}^{n}$,

$$
\gamma(u, X)=(\gamma u, \rho(\gamma) X) .
$$

Let $\left(G_{t}\right)_{t \in \mathbb{R}}$ be the flow on the total space $T^{1} S \times_{\rho} \mathbb{R}^{n}$ that lifts the geodesic flow $\left(g_{t}\right)_{t \in \mathbb{R}}$ on $T^{1} S$ via the flat connection; here, the "flatness" condition means that, if one looks at the situation in the universal cover, the lift $\left(\widetilde{G}_{t}\right)_{t \in \mathbb{R}}$ acts on $T^{1} \widetilde{S} \times \mathbb{R}^{n}$ as the geodesic flow $(\widetilde{g})_{t \in \mathbb{R}}$ on the first factor, and trivially on the second factor. We shall refer to $T^{1} S \times_{\rho} \mathbb{R}^{n} \rightarrow T^{1} S$ as the associated, flat $\mathbb{R}^{n}$-bundle of the Hitchin representation $\rho: \pi_{1}(S) \rightarrow \mathrm{PSL}_{n}(\mathbb{R})$.

For every nontrivial $\gamma \in \pi_{1}(S)$, index the eigenvalues $\lambda_{i}^{\rho}(\gamma)$ of $\rho(\gamma) \in \operatorname{PSL}_{n}(\mathbb{R})$ as in Theorem 2 so that

$$
\left|\lambda_{1}^{\rho}(\gamma)\right|>\left|\lambda_{2}^{\rho}(\gamma)\right|>\cdots>\left|\lambda_{n}^{\rho}(\gamma)\right|
$$

The key tool underlying Labourie's analysis is the following decomposition.

Theorem 6 (Labourie [19] eigenbundle decomposition) The associated, flat $\mathbb{R}^{n}$ bundle $p: T^{1} S \times{ }_{\rho} \mathbb{R}^{n} \rightarrow T^{1} S$ splits as a sum of $n$ line subbundles $V_{1} \oplus \cdots \oplus V_{n}$ that satisfy the following properties:

(i) Each line subbundle $V_{i} \rightarrow T^{1} S$ is invariant under the flow $\left(G_{t}\right)_{t \in \mathbb{R}}$.

(ii) If $u \in T^{1} S$ is fixed by $g_{t_{0}}: T^{1} S \rightarrow T^{1} S$ for some $t_{0}>0$, and if $\gamma \in \pi_{1}(S)$ represents the corresponding closed orbit of the geodesic flow, then the lift $G_{t_{0}}$ acts on the fibre $p^{-1}(u)=V_{1}(u) \oplus \cdots \oplus V_{n}(u)$ by multiplication by $1 / \lambda_{i}^{\rho}(\gamma)$ on the line $V_{i}(u)$.

(iii) Each line $V_{i}(u)$ depends smoothly on $u \in T^{1} S$ along the leaves of the geodesic foliation $\mathcal{F}$, and is transversally Hölder continuous. 
The terminology eigenbundle decomposition is motivated by the property (ii) that we can make more precise as follows. For every $i=1, \ldots, n$, let $\widetilde{V}_{i} \rightarrow T^{1} \widetilde{S}$ that lifts the line subbundle $V_{i} \rightarrow T^{1} S$. Let $\left(\widetilde{g}_{t}\right)_{t \in \mathbb{R}}$ be the lift on the universal cover $T^{1} \widetilde{S}$ of the geodesic flow $\left(g_{t}\right)_{t \in \mathbb{R}}$ on $T^{1} S$. Let $u \in T^{1} S$ that is fixed by $g_{t_{0}}: T^{1} S \rightarrow T^{1} S$ for some $t_{0}>0$. Let $\tilde{u} \in T^{1} \widetilde{S}$ that lifts $u \in T^{1} S$, and let $\gamma \in \pi_{1}(S)$ be the (unique) nontrivial element such that $\tilde{g}_{t_{0}}(\tilde{u})=\gamma \tilde{u}$. Because of the flat connection, and the invariance of the line subbundle $V_{i} \rightarrow T^{1} S$ under the flow $\left(G_{t}\right)_{t \in \mathbb{R}}$, we have that $\widetilde{V}_{i}\left(\widetilde{g}_{t_{0}}(\widetilde{u})\right)=\widetilde{V}_{i}(\widetilde{u})$ as lines of $\mathbb{R}^{n}$. Also, $\widetilde{V}_{i}\left(\widetilde{g}_{t_{0}}(\widetilde{u})\right)=\widetilde{V}_{i}(\gamma \tilde{u})=\rho(\gamma) \widetilde{V}_{i}(\widetilde{u})$ (it is the equivariance property for the lift $\left.\widetilde{V}_{i} \rightarrow T^{1} \widetilde{S}\right)$. Hence $\widetilde{V}_{i}(\widetilde{u}) \subset \mathbb{R}^{n}$ is an eigenspace for $\rho(\gamma) \in \operatorname{PSL}_{n}(\mathbb{R})$, and $\rho(\gamma)$ is diagonalizable. Finally, note that, for every $X \in \mathbb{R}^{n}$, $\left(\widetilde{g}_{t_{0}}(\tilde{u}), \widetilde{G}_{t_{0}} X\right)=(\gamma \tilde{u}, X)$ identifies in the quotient with $\left(\tilde{u}, \rho(\gamma)^{-1} X\right)$. Therefore, the lift $G_{t_{0}}$ acts on the line $V_{i}(u)$ by multiplication by $1 / \lambda_{i}^{\rho}(\gamma)$.

As a consequence of the above discussion, we make the following observation, that we state as a lemma for future reference.

Lemma 7 Let $\widetilde{V}_{i} \rightarrow T^{1} \widetilde{S}$ and $\widetilde{V}_{n-i+1} \rightarrow T^{1} \widetilde{S}$ that lift the line subbundles $V_{i} \rightarrow$ $T^{1} S$ and $V_{n-i+1} \rightarrow T^{1} S$, respectively. For every $u \in T^{1} S$ that lifts to $\tilde{u} \in T^{1} \widetilde{S}$, the fibres $\widetilde{V}_{i}(\widetilde{u})$ and $\widetilde{V}_{n-i+1}(-\widetilde{u})$ coincide as lines of $\mathbb{R}^{n}$.

Proof When $u$ lies in a closed leaf of the geodesic foliation $\mathcal{F}$, the assertion immediately comes as a consequence of the property (ii) of Theorem 6 , and from our indexing conventions for the eigenvalues of $\rho(\gamma) \in \operatorname{PSL}_{n}(\mathbb{R})$ when $\gamma \in \pi_{1}(S)$. The general case then follows from the latter by density of closed leaves in $T^{1} S$.

The existence of an eigenbundle decomposition for the associated, flat $\mathbb{R}$-bundle $T^{1} S \times{ }_{\rho} \mathbb{R}^{n} \rightarrow T^{1} S$ of a Hitchin representation $\rho: \pi_{1}(S) \rightarrow \mathrm{PSL}_{n}(\mathbb{R})$ as in Theorem 6 is a consequence of Labourie's Anosov Property for Hitchin representations; see Labourie [19], Guichard [16], Guichard and Wienhard [17] and Dreyer [9] for additional details.

\section{The length functions of a Hitchin representation}

\subsection{Hölder geodesic currents}

Before tackling the construction of the length functions $\ell_{i}^{\rho}: \mathcal{C}^{\text {Höl }}(S) \rightarrow \mathbb{R}$, we need to remind the reader of the definition of Hölder geodesic currents; see Bonahon $[2 ; 3 ; 4]$ for details.

Let $(X, d)$ be metric space. A Hölder distribution $\alpha$ is a continuous, linear functional on the space of compactly supported, Hölder continuous functions $\phi: X \rightarrow \mathbb{R}$. A 
special case of Hölder distributions are positive Radon measures, which are linear functionals on the space of compactly supported, continuous functions, and associate to a nonnegative function a nonnegative number.

The unit tangent bundle $T^{1} S$ is a 3 -dimensional manifold, and the orbits of the $m_{0}$-geodesic flow $\left(g_{t}\right)_{t \in \mathbb{R}}$ define a 1 -dimensional foliation $\mathcal{F}$ of $T^{1} S$ called its $m_{0}-$ geodesic foliation. It turns out that, whereas the geodesic flow depends of the auxiliary metric $m_{0}$ that we have chosen on $S$, the geodesic foliation does not. Indeed, if another negatively curved metric $m^{\prime}$ defines a geodesic foliation $\mathcal{F}^{\prime}$, there is a homeomorphism of $T^{1} S$ that sends $\mathcal{F}$ to $\mathcal{F}^{\prime}$. In addition, this homeomorphism can be chosen to be isotopic to the identity, and Hölder bicontinuous; see Bridson and Haefliger [7], Ghys [12] and Gromov [14; 15] for details.

A Hölder geodesic current $\alpha$ on $S$ is a transverse Hölder distribution for the geodesic foliation $\mathcal{F}$, namely $\alpha$ assigns a Hölder distribution $\alpha_{D}$ on every surface $D \subset T^{1} S$ transverse to $\mathcal{F}$. This assignment is invariant under restriction: for any subsurface $D^{\prime} \subset D,\left.\alpha_{D}\right|_{D^{\prime}}=\alpha_{D^{\prime}}$, and is homotopy invariant: for any (Hölder) homotopy $h: D \rightarrow D^{\prime \prime}$ from $D$ to another transverse surface $D^{\prime \prime}$ that preserves $\mathcal{F}, \alpha_{D}=h^{*} \alpha_{D^{\prime \prime}}$; $h^{*} \alpha_{D^{\prime \prime}}$ is the pullback of $\alpha_{D^{\prime \prime}}$ by $h$.

When the transverse Hölder distribution $\alpha$ is actually a measure $\alpha_{D}$ for every surface $D \subset T^{1} S$ transverse to $\mathcal{F}$, the corresponding Hölder geodesic current is a measure geodesic current of $S$. Let $\mathcal{C}^{\mathrm{Höl}}(S)$ and $\mathcal{C}(S)$ be respectively the space of Hölder geodesic currents, and the space of measure geodesic currents. Note that $\mathcal{C}^{\text {Höl }}(S)$ is a (real) vector space, and $\mathcal{C}(S)$ is stable under positive scalar multiplication.

The space of Hölder geodesic currents $\mathcal{C}^{\mathrm{Höl}}(S)$ is endowed with the weak-* topology, namely the weakest topology for which, for every surface $D \subset T^{1} S$ transverse to $\mathcal{F}$, the linear function $\varphi_{D} \mapsto \alpha_{D}\left(\varphi_{D}\right)$ is continuous, where $\varphi_{D}$ ranges over all compactly supported, Hölder continuous functions on the surface $D$.

A typical example of measure geodesic current is provided by the free homotopy class of a closed, oriented curve $\gamma \subset S$. Let $k \geq 0$ be the largest integer such that $\gamma$ is homotopic to a $k$-multiple $\gamma_{1}^{k}$ of a closed curve $\gamma_{1}$. The homotopically primitive curve $\gamma_{1}$ is freely homotopic to a unique closed, oriented geodesic, which itself corresponds to a closed leaf $\gamma_{1}^{*}$ of the geodesic foliation $\mathcal{F}$. In particular, we associate to $\gamma_{1}$ the transverse 1 -weighted Dirac measure for $\mathcal{F}$ defined by the closed orbit $\gamma_{1}^{*}$ : for every surface $D$ transverse to $\mathcal{F}$, the measure $\gamma_{1 D}$ is the counting measure at the intersection points $D \cap \gamma_{1}^{*}$. As a result, we associate to $\gamma=\gamma_{1}^{k} k$-times the transverse 1 -weighted Dirac measure associated to $\gamma_{1}$. Hence the following embedding

$\{$ closed, oriented curves in $S\} /$ homotopy $\subset \mathcal{C}(S) \subset \mathcal{C}^{\text {Höl }}(S)$. 
In addition, the set of positive real, linear combinations of multiples of homotopy classes of closed, oriented curves are dense in $\mathcal{C}(S)$.

Finally, note that to a closed, unoriented curve $\bar{\gamma}$ in $S$ corresponds two closed leaves $\gamma^{*}$ and $(\Re(\gamma))^{*}$ of the geodesic foliation $\mathcal{F}$, and thus two measure geodesic currents $\gamma$ and $\mathfrak{R}^{*} \gamma \in \mathcal{C}(S)$ (as in Theorem 3, $\mathfrak{R}: T^{1} S \rightarrow T^{1} S$ denotes the orientation reversing involution, and $\mathfrak{R}^{*}: \mathcal{C}^{\text {Höl }}(S) \rightarrow \mathcal{C}^{\text {Höl }}(S)$ is the pullback involution induced by $\left.\mathfrak{R}\right)$. Therefore, the set of closed, unoriented curves in $S$ can be formally embedded in $\mathcal{C}^{\text {Höl }}(S)$ as follows: for every closed, unoriented curve $\bar{\gamma} \subset S$,

$$
\bar{\gamma}=\frac{1}{2} \gamma+\frac{1}{2} \mathfrak{R}^{*} \gamma \in \mathcal{C}^{\mathrm{Höl}}(S) .
$$

In particular, the space of measured laminations $\mathcal{M L}(S)$, that is defined as the closure in $\mathcal{C}(S)$ of the set of positive real multiples of homotopy classes of simple, closed, unoriented curves in $S$ (Bonahon [1;2]), corresponds to the closure in $\mathcal{C}(S)$ of the set of positive real, linear combinations of elements of the above form (1).

\subsection{Lengths of closed, oriented curves}

Let $\rho: \pi_{1}(S) \rightarrow \operatorname{PSL}_{n}(\mathbb{R})$ be a Hitchin representation. For a nontrivial $\gamma \in \pi_{1}(S)$, Theorem 1 shows that the eigenvalues $\lambda_{i}^{\rho}(\gamma)$ of the matrix $\rho(\gamma) \in \operatorname{PSL}_{n}(\mathbb{R})$ are all real, and can be indexed so that

$$
\left|\lambda_{1}^{\rho}(\gamma)\right|>\left|\lambda_{2}^{\rho}(\gamma)\right|>\cdots>\left|\lambda_{n}^{\rho}(\gamma)\right| .
$$

Set $\ell_{i}^{\rho}(\gamma)=\log \left|\lambda_{i}^{\rho}(\gamma)\right|$. Note that $\ell_{i}^{\rho}(\gamma)$ depends only on the conjugacy class of $\gamma \in \pi_{1}(S)$, and thus depends only on the free homotopy class of the closed, oriented curve $\gamma \subset S$. We have $n$ maps

$$
\ell_{i}^{\rho}:\{\text { closed, oriented curves in } S\} / \text { homotopy } \rightarrow \mathbb{R} .
$$

\subsection{1-forms along the geodesic foliation}

We now construct, for every $i=1, \ldots, n$, a 1 -form $\omega_{i}$ along the leaves of the geodesic foliation $\mathcal{F}$ of $T^{1} S$.

Given a Hitchin representation $\rho: \pi_{1}(S) \rightarrow \operatorname{PSL}_{n}(\mathbb{R})$, consider its associated flat $\mathbb{R}^{n}$ bundle $T^{1} S \times{ }_{\rho} \mathbb{R}^{n} \rightarrow T^{1} S$ as in Section 1. Let $\left(G_{t}\right)_{t \in \mathbb{R}}$ the flow on $T^{1} S \times{ }_{\rho} \mathbb{R}^{n}$ that lifts the geodesic flow on $T^{1} S$ via the flat connection. Let $V_{1}, V_{2}, \ldots, V_{n} \rightarrow T^{1} S$ be the line subbundles of the eigenbundle decomposition of Theorem 6; a fundamental property for each of the line subbundles $V_{i} \rightarrow T^{1} S$ is to be invariant under the action of the flow $\left(G_{t}\right)_{t \in \mathbb{R}}$. Finally, pick a Riemannian metric \|\| on $T^{1} S \times_{\rho} \mathbb{R}^{n} \rightarrow T^{1} S$. 
Let $\mathcal{L}$ be a leaf of the geodesic foliation $\mathcal{F}$. Pick a point $u_{0} \in \mathcal{L} \subset T^{1} S$ and a vector $X_{i}\left(u_{0}\right)$ in the fibre $V_{i}\left(u_{0}\right)$ of the line subbundle $V_{i} \rightarrow T^{1} S$. For every $t$ on a neighborhood of 0 in $\mathbb{R}$, set

$$
f_{X_{i}\left(u_{0}\right)}\left(g_{t}\left(u_{0}\right)\right)=\log \left\|G_{t} X_{i}\left(u_{0}\right)\right\|_{g_{t}\left(u_{0}\right)} .
$$

The above expression defines a function $f_{X_{i}\left(u_{0}\right)}: I \rightarrow \mathbb{R}$, where $I$ is a neighborhood of $u_{0}$ in the leaf $\mathcal{L}$. In addition, the fibre $V_{i}(u)$ depending smoothly on the point $u \in T^{1} S$ along the leaves of the geodesic foliation $\mathcal{F}$, the function $f_{X_{i}\left(u_{0}\right)}$ is smooth along the leaf $\mathcal{L}$. For every $u$ on the same neighborhood $I$ of $u_{0}$ in the leaf $\mathcal{L}$, set

$$
\omega_{i}(u)=-d_{u} f_{X_{i}\left(u_{0}\right)},
$$

where the differential $d_{u} f_{X_{i}\left(u_{0}\right)}$ is taken along the leaf $\mathcal{L}$.

Lemma 8 Defined as above, $\omega_{i}$ is a well-defined 1 -form along the leaf $\mathcal{L} \subset \mathcal{F}$.

Proof We must verify that $\omega_{i}$ does not depend on the choices of the point $u_{0} \in I$, and of the vector $X_{i}\left(u_{0}\right) \in V_{i}\left(u_{0}\right)$.

Since the fibre $V_{i}\left(u_{0}\right)$ is a line, any other choice $X_{i}^{\prime}\left(u_{0}\right)$ for $X_{i}\left(u_{0}\right)$ is of the form $X_{i}^{\prime}\left(u_{0}\right)=c X_{i}\left(u_{0}\right)$ for some $c \in \mathbb{R}$. Then

$$
f_{X_{i}^{\prime}\left(u_{0}\right)}=f_{X_{i}\left(u_{0}\right)}+\log |c|
$$

and $d f_{X_{i}^{\prime}\left(u_{0}\right)}=d f_{X_{i}\left(u_{0}\right)}$. Hence $\omega_{i}$ is independent of the choice of $X_{i}\left(u_{0}\right) \in V_{i}\left(u_{0}\right)$. Let $u_{0}^{\prime}=g_{t_{0}}\left(u_{0}\right) \in I$ be another point, and let $X_{i}^{\prime}\left(u_{0}^{\prime}\right)=G_{t_{0}} X_{i}\left(u_{0}\right) \in V_{i}\left(u_{0}^{\prime}\right)$. Then the functions

$$
f_{u_{0}^{\prime}, X_{i}^{\prime}\left(u_{0}^{\prime}\right)}=f_{u_{0}, X_{i}\left(u_{0}\right)}
$$

coincide on $I$ since $G_{t} X_{i}^{\prime}\left(u_{0}^{\prime}\right)=G_{t+t_{0}} X_{i}\left(u_{0}\right)$, and thus have the same differential along the leaf $\mathcal{L}$.

It follows from Lemma 8 that $\omega_{i}$ is a well-defined 1 -form along the leaves of $\mathcal{F}$. Moreover, let us also make the following observation regarding the global regularity of the 1 -form $\omega_{i}$.

Lemma 9 The 1 -form $\omega_{i}$ is smooth along the leaves of the geodesic foliation $\mathcal{F}$, and is transversally Hölder continuous.

Proof This is an immediate consequence of the regularity property (iii) of Theorem 6 . 


\subsection{Lengths of Hölder geodesic currents}

We now make use of the 1 -forms $\omega_{i}$ to define the lengths $\ell_{i}^{\rho}(\alpha)$ of a Hölder geodesic current $\alpha \in \mathcal{C}^{\mathrm{Höl}}(S)$.

Let $\left\{\mathcal{U}_{j}\right\}_{j=1, \ldots, m}$ be a finite family of flow boxes $\left\{\mathcal{U}_{j}\right\}_{j=1, \ldots, m}$ that covers the compact, foliated 3-manifold $T^{1} S$. By flow box, we mean an open subset $\mathcal{U}_{j} \subset T^{1} S$ such that there exists a diffeomorphism $\mathcal{U}_{j} \cong D_{j} \times(0,1)$, where $D_{j}$ is an open subset of $\mathbb{R}^{2}$, and where, for every $x \in D_{j}$, the interval $\{x\} \times(0,1)$ corresponds to an arc in a leaf of $\mathcal{F}$. Let $\left\{\xi_{j}\right\}_{j=1, \ldots, m}$ be a partition of unity subordinate to the open covering $\left\{\mathcal{U}_{j}\right\}_{j=1, \ldots, m}$. By integrating the 1 -form $\xi_{j} \omega_{i}$ along the arcs of leaves in $\mathcal{U}_{j}$, we define a function $\phi_{j}: D_{j} \rightarrow \mathbb{R}$ by

$$
\phi_{j}(x)=\int_{\{x\} \times(0,1)} \xi_{j} \omega_{i} .
$$

Note that, by Lemma 9, the above function $\phi_{j}: D_{j} \rightarrow \mathbb{R}$ is Hölder continuous with compact support. The Hölder geodesic current $\alpha$ induces a Hölder distribution $\alpha_{D_{j}}$ on $D_{j}$, that we shall still denote by $\alpha$ to alleviate notations. Let us denote the evaluation of $\alpha$ at the function $\phi_{j}$ by

$$
\alpha\left(\phi_{j}\right)=\int_{\mathcal{U}_{j}} \xi_{j} \omega_{i} d \alpha
$$

where the integral notation is suggested by the case where $\alpha$ is a transverse measure for $\mathcal{F}$. Finally, set

$$
\ell_{i}^{\rho}(\alpha)=\int_{T^{1} S} \omega_{i} d \alpha=\sum_{j=1}^{m} \int_{\mathcal{U}_{j}} \xi_{j} \omega_{i} d \alpha .
$$

By the usual linearity arguments, $\ell_{i}^{\rho}(\alpha)$ is independent of the choice of the open covering $\left\{\mathcal{U}_{j}\right\}_{j=1, \ldots, m}$ and of the partition of unity $\left\{\xi_{j}\right\}_{j=1, \ldots, m}$.

Theorem 10 Defined as above, for every $i=1, \ldots, n$,

$$
\ell_{i}^{\rho}: \mathcal{C}^{\mathrm{Höl}}(S) \rightarrow \mathbb{R}
$$

is a continuous, linear function on the vector space of Hölder geodesic currents $\mathcal{C}^{\text {Höl }}(S)$ that extends the length $\ell_{i}^{\rho}$ of closed, oriented curves of Section 2.2. This continuous extension is unique on the space of measure geodesic currents $\mathcal{C}(S) \subset \mathcal{C}^{\mathrm{Höl}}(S)$. In addition, the length $\ell_{i}^{\rho}$ does not depend on the choice of the Riemannian metric \| \| on the associated, flat $\mathbb{R}^{n}$-bundle $T^{1} S \times_{\rho} \mathbb{R}^{n} \rightarrow T^{1} S$ that defines the 1-form $\omega_{i}$ of Section 2.3. 


\section{Proof of Theorem 10}

We organize the proof into several steps.

Lemma 11 For every closed, oriented curve $\gamma \subset S$,

$$
\ell_{i}^{\rho}(\gamma)=\log \left|\lambda_{i}^{\rho}(\gamma)\right|,
$$

where $\ell_{i}^{\rho}(\gamma)$ is the image of the Hölder geodesic current $\gamma \in \mathcal{C}^{\text {Höl }}(S)$ under the function $\ell_{i}^{\rho}: \mathcal{C}^{\mathrm{Holl}}(S) \rightarrow \mathbb{R}$, and where $\lambda_{i}^{\rho}(\gamma)$ is the $i^{\text {th }}$ eigenvalue of $\rho(\gamma)$.

Proof We need to return to the definition of the Hölder geodesic current $\gamma \in \mathcal{C}^{\mathrm{Höl}}(S)$.

By homogeneity of the function $\ell_{i}^{\rho}$, we can focus attention to the case where the closed, oriented curve $\gamma \subset S$ is homotopically primitive, namely $\gamma$ is not homotopic to a multiple $\gamma_{1}^{k}$ of a closed, oriented curve $\gamma_{1}$ with $k \geq 2$. Thus $\gamma$ determines a closed, oriented $m_{0}$-geodesic of $S$, and a closed leaf $\gamma^{*}$ of the geodesic foliation $\mathcal{F}$. Identify the closed, oriented curve $\gamma \subset S$ with the Hölder geodesic current $\gamma \in \mathcal{C}^{\text {Höl }}(S)$, which is the transverse 1-weighted Dirac measure defined by the associated closed leaf $\gamma^{*}$ (see Section 2.1).

By definition of the function $\ell_{i}^{\rho}: \mathcal{C}^{\mathrm{Höl}}(S) \rightarrow \mathbb{R}$,

$$
\ell_{i}^{\rho}(\gamma)=\int_{T^{1} S} \omega_{i} d \gamma=\int_{\gamma^{*}} \omega_{i}
$$

To compute this integral, pick a point $u_{0} \in \gamma^{*} \subset T^{1} S$, and a nonzero vector $X_{i}\left(u_{0}\right) \in$ $V_{i}\left(u_{0}\right)$ in the fibre of the line subbundle $V_{i} \rightarrow T^{1} S$. By definition of the 1-form $\omega_{i}$ (see Section 2.3),

$$
\begin{aligned}
\int_{\gamma^{*}} \omega_{i} & =\int_{0}^{t_{\gamma}} \frac{d}{d s}\left(-\log \left\|G_{s} X_{i}\left(u_{0}\right)\right\|_{g_{s}\left(u_{0}\right)}\right) d s \\
& =\log \left\|G_{0} X_{i}\left(u_{0}\right)\right\|_{u_{0}}-\log \left\|G_{t_{\gamma}} X_{i}\left(u_{0}\right)\right\|_{g_{t_{\gamma}}\left(u_{0}\right)},
\end{aligned}
$$

where $t_{\gamma}$ is the necessary time to go around the closed leaf $\gamma^{*} \subset \mathcal{F}$ by the geodesic flow $\left(g_{t}\right)_{t \in \mathbb{R}}$, namely $t_{\gamma}$ is the smallest $t>0$ such that $g_{t}\left(u_{0}\right)=u_{0}$. By the property (ii) of Theorem $6, G_{t_{\gamma}} X_{i}\left(u_{0}\right)=1 / \lambda_{i}^{\rho}(\gamma) X_{i}\left(u_{0}\right)$, and $G_{0} X_{i}\left(u_{0}\right)=X_{i}\left(u_{0}\right)$ since $\left(G_{t}\right)_{t \in \mathbb{R}}$ is a flow, which proves the assertion.

Lemma 12 The function $\ell_{i}^{\rho}: \mathcal{C}^{\mathrm{Höl}}(S) \rightarrow \mathbb{R}$ is linear and continuous. Its restriction $\ell_{i}^{\rho} \mid \mathcal{C}(S): \mathcal{C}(S) \rightarrow \mathbb{R}$ is positively homogeneous, and is the unique, continuous extension to the space of measure geodesic currents $\mathcal{C}(S) \subset \mathcal{C}^{\mathrm{Höl}}(S)$ for the length $\ell_{i}^{\rho}$ of closed, oriented curves of Section 2.2. 
Proof By construction, for every Hölder geodesic current $\alpha \in \mathcal{C}^{\text {Höl }}(S)$,

$$
\ell_{i}^{\rho}(\alpha)=\sum_{j=1}^{m} \alpha\left(\phi_{j}\right)
$$

The linearity and homogeneity are immediate. The continuity follows from the definition of the weak-* topology of $\mathcal{C}^{\text {Höl }}(S)$. Finally, since the set of positive real, linear combinations of multiples of closed, oriented curves are dense in the space of measure geodesic currents $\mathcal{C}(S)$, the restriction of this continuous extension to $\mathcal{C}(S)$ is unique.

Lemma 13 The length $\ell_{i}^{\rho}(\alpha)$ is independent of the choice of the Riemannian metric \|\| on the associated, flat $\mathbb{R}^{n}$-bundle $T^{1} S \times{ }_{\rho} \mathbb{R}^{n} \rightarrow T^{1} S$.

Proof Let \|\|$^{\prime}$ be another Riemannian metric on the associated, flat $\mathbb{R}^{n}$-bundle $T^{1} S \times{ }_{\rho} \mathbb{R}^{n}$; it induces another 1 -form $\omega_{i}^{\prime}$ along the leaves of the geodesic foliation $\mathcal{F}$. Since the line $V_{i}(u)$ depends smoothly on $u \in T^{1} S$ along the leaves of $\mathcal{F}$, there exists a positive function $f: T^{1} S \rightarrow \mathbb{R}$, smooth along of the leaves of $\mathcal{F}$, such that, for every $X_{i}(u) \in V_{i}(u),\left\|X_{i}(u)\right\|_{u}=f(u)\left\|X_{i}(u)\right\|_{u}^{\prime}$. As a result, $\omega_{i}^{\prime}=\omega_{i}-d \log f$, which implies that

$$
\int_{T^{1} S} \omega_{i}^{\prime} d \alpha=\int_{T^{1} S} \omega_{i} d \alpha-\int_{T^{1} S} d \log f d \alpha
$$

Since $\sum_{j=1}^{m} \xi_{j}=1$,

$$
\int_{T^{1} S} d \log f d \alpha=\sum_{j=1}^{m} \int_{\mathcal{U}_{j}} d\left(\xi_{j} \log f\right) d \alpha .
$$

For our notation conventions, for every $j=1, \ldots, m$,

$$
\int_{\mathcal{U}_{j}} d\left(\xi_{j} \log f\right) d \alpha=\alpha_{D_{j}} \int_{\{x\} \times(0,1)} d\left(\xi_{j} \log f\right),
$$

where $\mathcal{U}_{j} \cong D_{j} \times(0,1)$. By Stokes's, $\int_{\{x\} \times(0,1)} d\left(\xi_{j} \log f\right)=0$, which proves the assertion.

This achieves the proof of Theorem 10. 


\section{Properties of the length functions}

\subsection{Symmetries of the lengths}

We now prove the two properties of Theorem 3 .

Proposition 14 For every Hölder geodesic current $\alpha \in \mathcal{C}^{\mathrm{Höl}}(S)$,

$$
\sum_{i=1}^{n} \ell_{i}^{\rho}(\alpha)=0
$$

Proof Endow each fibre $\{\widetilde{u}\} \times \mathbb{R}^{n}$ of the trivial bundle $T^{1} \widetilde{S} \times \mathbb{R}^{n} \rightarrow T^{1} \widetilde{S}$ with the canonical volume form $\sigma=d x_{1} \wedge d x_{2} \wedge \cdots \wedge d x_{n}$ of $\mathbb{R}^{n}$. Recall that $\pi_{1}(S)$ acts on $T^{1} \widetilde{S} \times \mathbb{R}^{n}$ via the diagonal action. Since each $\rho(\gamma)$ is in $\operatorname{SL}_{n}(\mathbb{R})$, the form $\sigma$ is invariant under the action of $\pi_{1}(S)$. In addition, because of the flat connection, the lift $\left(\widetilde{G}_{t}\right)_{t \in \mathbb{R}}$ on $T^{1} \widetilde{S} \times \mathbb{R}^{n}$ of the geodesic flow $\left(\widetilde{g}_{t}\right)_{t \in \mathbb{R}}$ on $T^{1} \widetilde{S}$ acts trivially on the factor $\mathbb{R}^{n}$ of $T^{1} \widetilde{S} \times \mathbb{R}^{n}$, and consequently preserves $\sigma$. As a result, $\sigma$ descends to a well-defined $G_{t}$-invariant volume form on the fibres of the bundle $T^{1} S \times_{\rho} \mathbb{R}^{n}$.

Recall that the length functions $\ell_{i}^{\rho}: \mathcal{C}^{\text {Höl }}(S) \rightarrow \mathbb{R}$ are independent of the choice of the Riemannian metric \|\| on the bundle $T^{1} S \times{ }_{\rho} \mathbb{R}^{n}$. Without loss of generality, we can arrange that the line subbundles $V_{i}$ are orthogonal for \|\| , and that the volume form defined by \|\| coincides with the volume form $\sigma$.

By definition of the 1 -form $\omega_{i}$, for every $u \in T^{1} S$, for every vector $X_{i}(u) \in V_{i}(u)$,

$$
\begin{aligned}
\sum_{i=1}^{n} \omega_{i}(u) & =\left.\sum_{i=1}^{n} \frac{d}{d t}\left(-\log \left\|G_{t} X_{i}(u)\right\|_{g_{t}(u)}\right) d t\right|_{t=0} \\
& =-\left.\frac{d}{d t} \log \left(\prod_{i=1}^{n}\left\|G_{t} X_{i}(u)\right\|_{g_{t}(u)}\right) d t\right|_{t=0} \\
& =-\left.\frac{d}{d t} \log \left(\sigma_{g_{t}(u)}\left(G_{t} X_{1}(u), G_{t} X_{2}(u), \ldots, G_{t} X_{n}(u)\right)\right) d t\right|_{t=0} \\
& =-\left.\frac{d}{d t} \log \left(\sigma_{u}\left(X_{1}(u), X_{2}(u), \ldots, X_{n}(u)\right)\right) d t\right|_{t=0}=0 .
\end{aligned}
$$

By integrating, it follows that, for every Hölder geodesic current $\alpha \in \mathcal{C}^{\text {Höl }}(S)$,

$$
\sum_{i=1}^{n} \ell_{i}^{\rho}(\alpha)=0
$$


The unit tangent space $T^{1} S$ comes endowed with a natural, fibrewise involution $\Re: T^{1} S \rightarrow T^{1} S$, which to $u \in T_{x}^{1} S$ associates $\mathfrak{R}(u)=-u$ : it is the orientation reversing involution. In particular, $\mathfrak{R}$ respects the geodesic foliation $\mathcal{F}$, and thus induces an involution $\mathfrak{R}^{*}: \mathcal{C}^{\mathrm{Höl}}(S) \rightarrow \mathcal{C}^{\text {Höl }}(S)$ defined as follows: for every Hölder geodesic current $\alpha \in \mathcal{C}^{\mathrm{Höl}}(S), \mathfrak{R}^{*} \alpha$ is the pullback current of $\alpha$ under the involution $\mathfrak{R}$, namely, if $\varphi: D \rightarrow \mathbb{R}$ is Hölder continuous with compact support defined on a transverse surface $D \subset T^{1} S$, then $\mathfrak{R}^{*} \alpha(\varphi)=\alpha(\varphi \circ \mathfrak{R})$. Note that the restriction to each oriented leaf of the geodesic foliation $\mathcal{F}$ of the involution $\mathfrak{R}$ is orientation reversing.

Proposition 15 For every Hölder geodesic current $\alpha \in \mathcal{C}^{\mathrm{Höl}}(S)$,

$$
\ell_{i}^{\rho}\left(\Re^{*} \alpha\right)=-\ell_{n-i+1}^{\rho}(\alpha) .
$$

Proof The involution $\mathfrak{R}$ acts freely on the unit tangent bundle $T^{1} S$ with quotient $\widehat{T}^{1} S=T^{1} S / \mathfrak{R}$. Thus, it also acts freely on the total space $T^{1} S \times_{\rho} \mathbb{R}^{n}$ with quotient a bundle $\widehat{T}^{1} S \times{ }_{\rho} \mathbb{R}^{n}$. Consider a Riemannian metric \|\| on $T^{1} S \times{ }_{\rho} \mathbb{R}^{n}$ obtained by lifting a Riemannian metric on $\widehat{T}^{1} S \times_{\rho} \mathbb{R}^{n}$. By construction, the Riemannian metric \|| $\|$ is invariant under the involution $\mathfrak{R}$.

On the other hand, let $\widetilde{V}_{i} \rightarrow T^{1} \widetilde{S}$ and $\widetilde{V}_{n-i+1} \rightarrow T^{1} \widetilde{S}$ that lift the subbundles $V_{i} \rightarrow T^{1} S$ and $V_{n-i+1} \rightarrow T^{1} S$, respectively. By Lemma 7 , for every $\tilde{u} \in T^{1} S$, for every $t \in \mathbb{R}$, the fibres $\widetilde{V}_{i}\left(\widetilde{g}_{t}(\Re(\widetilde{u}))\right)$ and $\widetilde{V}_{n-i+1}\left(\widetilde{g}_{-t}(\widetilde{u})\right)$ coincide as lines of $\mathbb{R}^{n}$. Therefore, $\mathfrak{R}^{*} \omega_{i}=\omega_{n-i+1}$. By integrating, it follows that, for every Hölder geodesic current $\alpha \in \mathcal{C}^{\mathrm{Höl}}(S), \ell_{i}^{\rho}\left(\mathfrak{R}^{*} \alpha\right)=-\ell_{n-i+1}^{\rho}(\alpha)$; note that a minus sign pops up due to the orientation reversing property of the involution $\mathfrak{R}$.

\subsection{Differentiability of the lengths}

We discuss some regularity properties for the length functions $\ell_{i}^{\rho}: \mathcal{C}^{\text {Höl }}(S) \rightarrow \mathbb{R}$.

Thurston [25] considers the space of measured laminations $\mathcal{M L}(S)$ of $S$, that is a certain completion of the set of all isotopy classes of simple, closed, unoriented curves in $S$. A fundamental feature of the space $\mathcal{M L}(S)$ is that it is a piecewise linear manifold, which is homeomorphic to $\mathbb{R}^{6 g-6}$. Therefore, every measured lamination admits tangent vectors, which allows to tackle tangentiality properties for functions that are defined on $\mathcal{M L}(S)$. We refer the reader to Penner and Harer [22] and Bonahon [3] for additional details about the PL-structure of $\mathcal{M L}(S)$.

The definition of tangent vectors to $\mathcal{M L}(S)$ is rather abstract and not very convenient in practice. In [3], Bonahon gives an analytical interpretation for the tangent vectors to 
$\mathcal{M L}(S)$ as certain Hölder geodesic currents. In particular, a consequence of this work is the following simple criterion.

Recall that the space of measured laminations $\mathcal{M L}(S)$ can be viewed as the closure in $\mathcal{C}^{\mathrm{Höl}}(S)$ of the set of positive real, linear combinations elements of the form

$$
\bar{\gamma}=\frac{1}{2} \gamma+\frac{1}{2} \mathfrak{R}^{*} \gamma,
$$

where $\gamma \in \mathcal{C}^{\text {Höl }}(S)$ is a closed, oriented curve, and $\mathfrak{R}: T^{1} S \rightarrow T^{1} S$ is the orientation reversing involution; see Section 2.1.

Theorem 16 (Bonahon [3]) Let $f: \mathcal{M L}(S) \rightarrow \mathbb{R}$ be a homogeneous function defined on the space of measured lamination $\mathcal{M L}(S)$. If $f$ admits a continuous, linear extension $f: \mathcal{C}^{\mathrm{Höl}}(S) \rightarrow \mathbb{R}$ to the vector space of Hölder geodesic currents $\mathcal{C}^{\text {Höl }}(S)$, then $f: \mathcal{M L}(S) \rightarrow \mathbb{R}$ is tangentiable, namely it is differentiable with respect to directions of tangent vectors to $\mathcal{M L}(S)$.

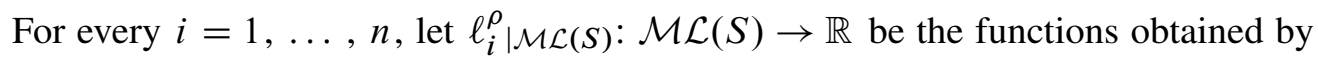
restricting the length functions $\ell_{i}^{\rho}: \mathcal{C}^{\mathrm{Höl}}(S) \rightarrow \mathbb{R}$ to the space of measured laminations $\mathcal{M L}(S)$. The following corollary is a straightforward consequence of the criterion of Theorem 16.

Corollary 17 The functions $\ell_{i}^{\rho} \mid \mathcal{M L}(S): \mathcal{M L}(S) \rightarrow \mathbb{R}$ are tangentiable, namely, if $\left(\alpha_{t}\right)_{t \geq 0} \subset \mathcal{M L}(S)$ is a smooth 1-parameter family of measured laminations with tangent vector $\dot{\alpha_{0}}=\left.\frac{d}{d t^{+}} \alpha_{t}\right|_{t=0}$ at $\alpha_{0}$, then

$$
\left.\frac{d}{d t^{+}} \ell_{i}^{\rho}\left(\alpha_{t}\right)\right|_{t=0}=\ell_{i}^{\rho}\left(\dot{\alpha}_{0}\right)
$$

\section{An asymptotic estimate for the eigenvalues}

As another application of the continuity property of the lengths $\ell_{i}^{\rho}$, we now prove the following estimate.

Theorem 18 Let $\rho: \pi_{1}(S) \rightarrow \mathrm{PSL}_{n}(\mathbb{R})$ be a Hitchin representation, and let $\alpha, \beta \in$ $\pi_{1}(S)$. For every $i=1, \ldots, n$, the ratio

$$
\frac{\lambda_{i}^{\rho}\left(\alpha^{m} \beta\right)}{\lambda_{i}^{\rho}(\alpha)^{m}}
$$

has a finite limit as $m$ tends to $\infty$. This limit is equal to $e^{\ell_{i}^{\rho}(\dot{\alpha})}$, where $\dot{\alpha}$ is the Hölder geodesic current $\dot{\alpha}=\lim _{m \rightarrow \infty} \alpha^{m} \beta-m \alpha \in \mathcal{C}^{\mathrm{Höl}}(S)$. 
Proof Without loss of generality, we can assume that $\alpha$ is primitive in $\pi_{1}(S)$. As in Section 2.1, identify the closed, oriented curves $\alpha$ and $\alpha^{m} \beta$ with the corresponding closed leaves $\alpha^{*}$ and $\alpha^{m} \beta^{*}$ of the geodesic foliation $\mathcal{F}$ of $T^{1} S$. Endowing these closed leaves with the transverse Dirac measures that they define, we can regard $\alpha$ and $\alpha^{m} \beta$ as Hölder geodesic currents.

For $m$ large enough, the closed leaf $\alpha^{m} \beta$ is made up of one piece of uniformly bounded length, and of another piece that wraps $m$ times around $\alpha$. As $m$ tends to $\infty$, this closed leaf converges to the union of the closed orbit $\alpha$ and of an infinite leaf $\alpha^{\infty} \beta$ of the geodesic foliation whose two ends spiral around $\alpha$; Figure 1 shows the situation in the surface $S$.
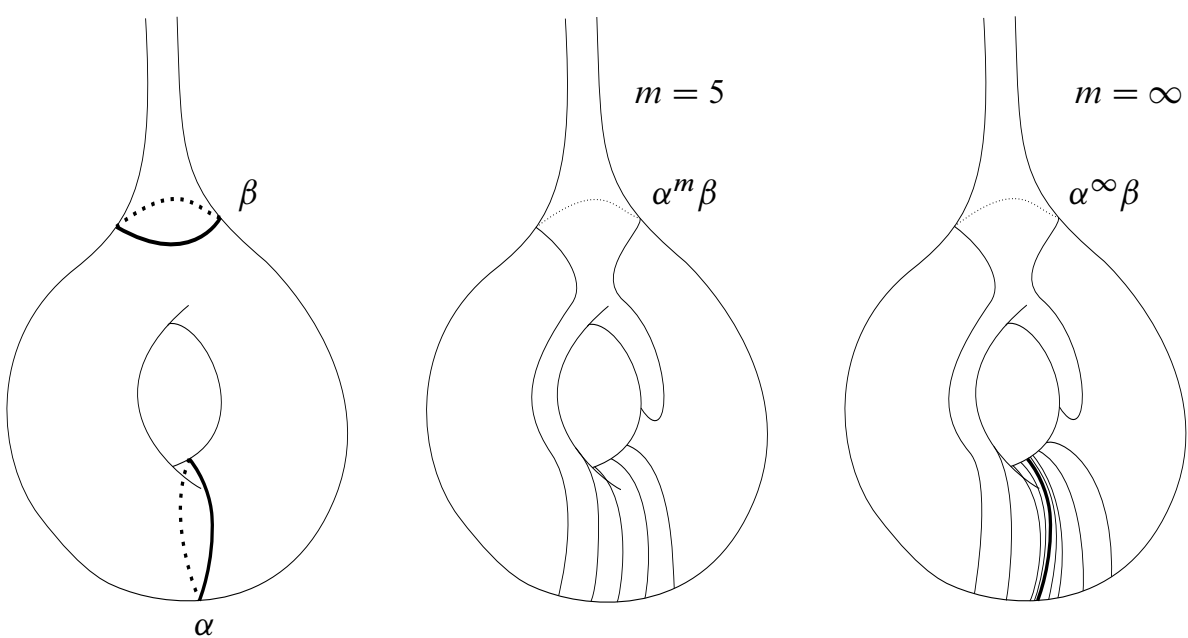

Figure 1: Projection of the leaf $\alpha \beta^{m}$ of the geodesic foliation $\mathcal{F}$ in the surface $S$

More precisely, let $D \subset T^{1} S$ be a small surface transverse to the geodesic foliation $\mathcal{F}$ that intersects the closed leaf $\alpha^{*}$ in one point $x_{\infty}^{\infty}$, as shown on Figure 2. The infinite leaf $\alpha^{\infty} \beta^{*}$ intersects $D$ in two sequences of points $x_{1}^{\infty}, x_{2}^{\infty}, \ldots$ and $y_{1}^{\infty}, y_{2}^{\infty}, \ldots$ in such a way that $x_{1}^{\infty}, x_{2}^{\infty}, \ldots$ converges in this order to one end of $\alpha^{\infty} \beta$, and $y_{1}^{\infty}, y_{2}^{\infty}, \ldots$ converges in this order to the other end. Moreover, the two sequences $x_{1}^{\infty}, x_{2}^{\infty}, \ldots$ and $y_{1}^{\infty}, y_{2}^{\infty}, \ldots$ both converge to the point $x_{\infty}^{\infty}$.

Likewise, the closed leaf $\alpha^{m} \beta^{*}$ intersects $D$ in points $x_{1}^{m}, x_{2}^{m}, \ldots, x_{k_{m}}^{m}, y_{1}^{m}, y_{2}^{m}, \ldots$, $y_{l_{m}}^{m}$ (see Figure 2), in such a way that, as $m$ tends to $\infty$, each $x_{k}^{m}$ converges to $x_{k}^{\infty}$, and each $y_{l}^{m}$ converges to $y_{l}^{\infty}$. Besides, the total number $k_{m}+l_{m}$ of points is of the order of $m$, and more precisely, the difference $m-\left(k_{m}+l_{m}\right)$ is equal to a constant 
$c_{D}$ for $m$ large enough. As a result, if $\varphi$ is a continuous function defined on $D$,

$$
\begin{aligned}
\lim _{m \rightarrow \infty} \frac{1}{m} \alpha^{m} \beta(\varphi)-\alpha & (\varphi) \\
& =\lim _{m \rightarrow \infty} \frac{1}{m}\left(\sum_{i=1}^{k_{m}} \varphi\left(x_{i}^{m}\right)+\sum_{j=1}^{l_{m}} \varphi\left(y_{j}^{m}\right)-\left(k_{m}+l_{m}+c_{D}\right) \varphi\left(x_{\infty}^{\infty}\right)\right) \\
& =0 .
\end{aligned}
$$
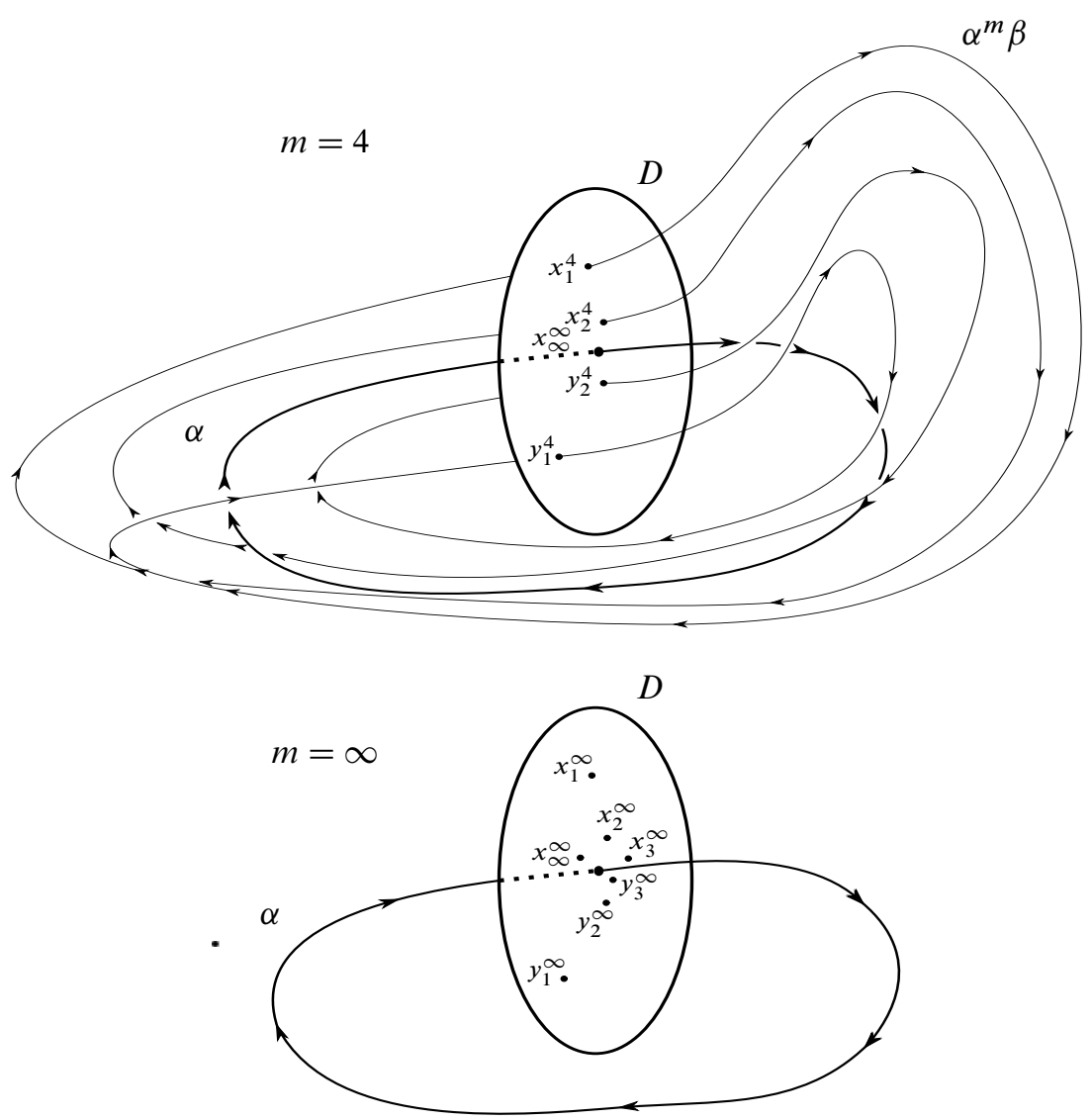

Figure 2: A transverse surface $D$ to the geodesic foliation $\mathcal{F}$ that intersects both leaves $\alpha$ and $\alpha \beta^{m}$

If, in addition, we assume $\varphi$ to be Hölder continuous, we can refine the above estimate. Because of the compacity of $S$, classical hyperbolic estimates guarantee that there exists some bound $M>0$ such that, for every $m \geq M$, both sequences $x_{i}^{m}$ and $y_{i}^{m}$ converge uniformly to $x_{\infty}^{\infty}$ as $i$ tends to $\infty$, and this convergence is of exponential 
order. Therefore

$$
\begin{aligned}
\lim _{m \rightarrow+\infty}\left(\alpha^{m} \beta-\right. & m \alpha)(\varphi) \\
& =\lim _{m \rightarrow+\infty} \sum_{i=1}^{k_{m}}\left(\varphi\left(x_{i}^{m}\right)-\varphi\left(x_{\infty}^{\infty}\right)\right)+\sum_{j=1}^{l_{m}}\left(\varphi\left(y_{j}^{m}\right)-\varphi\left(x_{\infty}^{\infty}\right)\right)-c_{D} \varphi\left(x_{\infty}^{\infty}\right) \\
& =\sum_{i=1}^{\infty}\left(\varphi\left(x_{i}^{\infty}\right)-\varphi\left(x_{\infty}^{\infty}\right)\right)+\sum_{j=1}^{\infty}\left(\varphi\left(y_{j}^{\infty}\right)-\varphi\left(x_{\infty}^{\infty}\right)\right)-c_{D} \varphi\left(x_{\infty}^{\infty}\right)
\end{aligned}
$$

exists and is finite.

In other words, the above calculation shows that the limit

$$
\lim _{m \rightarrow \infty} \alpha^{m} \beta-m \alpha=\dot{\alpha}
$$

exists in the space of Hölder geodesic currents $\mathcal{C}^{\text {Höl }}(S)$. The limit Hölder geodesic current $\dot{\alpha}$ is supported in the union of the closed leaf $\alpha^{*}$, and of the infinite leaf $\alpha^{\infty} \beta^{*}$ whose two ends spiral around $\alpha^{*}$.

Thus, by linearity and continuity of the length functions $\ell_{i}^{\rho}$,

$$
\lim _{m \rightarrow \infty} \ell_{i}^{\rho}\left(\alpha^{m} \beta\right)-m \ell_{i}^{\rho}(\alpha)
$$

exists and is equal to $\ell_{i}^{\rho}(\dot{\alpha})$. Taking the exponential on both sides, we conclude that

$$
\frac{\lambda_{i}^{\rho}\left(\alpha^{m} \beta\right)}{\lambda_{i}^{\rho}(\alpha)^{m}}
$$

converges to $\mathrm{e}^{\ell_{i}^{\rho}(\dot{\alpha})}$, which proves the required result.

We conclude this section with one last observation:

$$
\dot{\alpha}=\lim _{m \rightarrow \infty} \alpha^{m} \beta-m \alpha=\lim _{m \rightarrow \infty} \frac{\frac{1}{m} \alpha^{m} \beta-\alpha}{\frac{1}{m}}
$$

is very reminiscent of the expression of a derivative. In fact, there exists a 1-parameter family of Hölder geodesic currents $\alpha_{t} \in \mathcal{C}^{\mathrm{Höl}}(S), t \in[0, \varepsilon]$, such that $\alpha_{0}=\alpha, \alpha_{1 / m}=$ $\frac{1}{m} \alpha^{m} \beta$, and $\dot{\alpha}=\left.\frac{d}{d t^{+}} \alpha_{t}\right|_{t=0}$. As a result, the distribution $\dot{\alpha}$ should be understood as a tangent vector at the point $\alpha$, and the above estimate comes as a consequence of the first order approximation

$$
\ell_{i}^{\rho}\left(\alpha_{t}\right) \approx \ell_{i}^{\rho}\left(\alpha_{0}\right)+\left.t \frac{d}{d t^{+}} \ell_{i}^{\rho}\left(\alpha_{t}\right)\right|_{t=0},
$$


where $\left.\frac{d}{d t^{+}} \ell_{i}^{\rho}\left(\alpha_{t}\right)\right|_{t=0}=\ell_{i}^{\rho}(\dot{\alpha})$ by the previous facts. This should be compared with the differentiability property of the length functions $\ell_{i}^{\rho}$ of Corollary 17.

Remark In a preprint following the publication of an earlier version of the present paper, M Pollicott and R Sharp [23] proposed an alternative proof of the estimate of Theorem 18 that is based on symbolic dynamics. Their method enables to improve the result. They also prove asymptotic growth estimates for the length functions $\ell_{i}^{\rho}$. In particular, their approach makes crucially use of the 1 -forms $\omega_{i}$ introduced in Section 2.3.

\section{References}

[1] F Bonahon, Bouts des variétés hyperboliques de dimension 3, Ann. of Math. 124 (1986) 71-158 MR847953

[2] F Bonahon, The geometry of Teichmüller space via geodesic currents, Invent. Math. 92 (1988) 139-162 MR931208

[3] F Bonahon, Geodesic laminations with transverse Hölder distributions, Ann. Sci. École Norm. Sup. 30 (1997) 205-240 MR1432054

[4] F Bonahon, Transverse Hölder distributions for geodesic laminations, Topology 36 (1997) 103-122 MR1410466

[5] F Bonahon, G Dreyer, Hitchin representations and geodesic laminations, in preparation

[6] F Bonahon, G Dreyer, Parametrizing Hitchin components arXiv:1209.3526

[7] M R Bridson, A Haefliger, Metric spaces of non-positive curvature, Grundl. Math. Wissen. 319, Springer, Berlin (1999) MR1744486

[8] S Choi, W M Goldman, Convex real projective structures on closed surfaces are closed, Proc. Amer. Math. Soc. 118 (1993) 657-661 MR1145415

[9] G Dreyer, Thurston's cataclysms for Anosov representations arXiv:1209.3526

[10] A Fathi, F Laudenbach, V Poénaru, Thurston's work on surfaces, Mathematical Notes 48, Princeton Univ. Press (2012) MR3053012

[11] V Fock, A Goncharov, Moduli spaces of local systems and higher Teichmüller theory, Publ. Math. Inst. Hautes Études Sci. (2006) 1-211 MR2233852

[12] É Ghys, P de la Harpe, Editors, Sur les groupes hyperboliques d'après Mikhael Gromov, Progress in Mathematics 83, Birkhäuser, Boston, MA (1990)

[13] W M Goldman, Topological components of spaces of representations, Invent. Math. 93 (1988) 557-607 MR952283 
[14] M Gromov, Hyperbolic groups, from: "Essays in group theory", (S M Gernsten, editor), Math. Sci. Res. Inst. Publ. 8, Springer, New York (1987) 75-263 MR919829

[15] M Gromov, Three remarks on geodesic dynamics and fundamental group, Enseign. Math. 46 (2000) 391-402 MR1805410

[16] O Guichard, Composantes de Hitchin et représentations hyperconvexes de groupes de surface, J. Differential Geom. 80 (2008) 391-431 MR2472478

[17] O Guichard, A Wienhard, Anosov representations: domains of discontinuity and applications, Invent. Math. 190 (2012) 357-438 MR2981818

[18] NJ Hitchin, Lie groups and Teichmüller space, Topology 31 (1992) 449-473 MR1174252

[19] F Labourie, Anosov flows, surface groups and curves in projective space, Invent. Math. 165 (2006) 51-114 MR2221137

[20] G A Margulis, Discrete subgroups of semisimple Lie groups, Ergeb. Math. Grenzgeb. 17, Springer, Berlin (1991) MR1090825

[21] D Mumford, J Fogarty, F Kirwan, Geometric invariant theory, 3rd edition, Ergeb. Math. Grenzgeb. 34, Springer, Berlin (1994) MR1304906

[22] R C Penner, J L Harer, Combinatorics of train tracks, Annals of Mathematics Studies 125, Princeton Univ. Press (1992) MR1144770

[23] M Pollicott, R Sharp, Length asymptotics in higher Teichmuller theory, in preparation

[24] W P Thurston, Minimal stretch maps between hyperbolic surfaces arXiv:9801039

[25] W P Thurston, The geometry and topology of 3-manifolds, Princeton University (1982)

[26] A Weil, On discrete subgroups of Lie groups, Ann. of Math. 72 (1960) 369-384 MR0137792

Department of Mathematics, University of Notre Dame

255 Hurley Hall, Notre Dame, IN 46556, USA

dreyfactor@gmail.com

Received: 11 September 2012 Revised: 4 February 2013 